\title{
GARANTÍAS EXPLICITAS EN SALUD: CONOCIMIENTO Y FUENTES DE INFORMACIÓN EN UN GRUPO DE MUJERES DEL PROGRAMA AUGE CÁNCER CÉRVICO UTERINO
}

\author{
María Teresa Urrutia S. ${ }^{a}$, Natalia Villegas $R^{b}$, Lauren Poupin $B .{ }^{c}$ \\ a Enfermera Matrona, PhD, Facultad de Medicina, Escuela de Enfermería, Pontificia Universidad Católica de Chile. \\ b Enfermera Matrona, Magister en Enfermería. ${ }^{C}$ Enfermera Matrona, Magister en Salud Pública, Facultad de Medicina, \\ Escuela de Enfermería, Pontificia Universidad Católica de Chile.
}

Esta investigación forma parte del proyecto FONIS (Fondo Nacional de Investigación en Salud) SA05I20047, CONICYT, MINSAL, Chile.

\section{RESUMEN}

Antecedentes: El cáncer cérvicouterino (CC) es una patología incluida en el sistema AUGE por su alto impacto en la población. En este sistema, todo usuario tiene el derecho de estar informado sobre su funcionamiento para favorecer la atención oportuna, sin embargo, actualmente se desconoce el nivel de conocimientos de las usuarias. Objetivo: Identificar el grado de conocimiento y las fuentes de información sobre el sistema GES que tienen las mujeres atendidas en el programa CC-AUGE. Método: Estudio descriptivo transversal, con una muestra de 364 mujeres, 237 con lesiones preinvasoras y 127 con CC. Resultados: El $18,7 \%$ de las mujeres refiere conocer el sistema GES, $24,5 \%$ lo conoce parcialmente y $56,7 \%$ no lo conoce. El $62 \%$ de las mujeres entiende por GES como un sistema que otorga protección financiera y el $45 \%$ que cubre enfermedades graves o catastróficas. En cuanto a fuentes de información sobre las GES, $71 \%$ refiere que recibió la información por parte del personal de salud o servicio de salud, el $29 \%$ por medios de comunicación masiva, el $8 \%$ por vecinos o familiares y $8 \%$ otras fuentes. Conclusiones: El grado de conocimiento acerca del sistema GES es bajo en las mujeres y se relaciona principalmente con la protección financiera y cobertura de enfermedades. Se considera relevante mejorar la entrega de información a las usuarias para fortalecer el funcionamiento eficiente de este sistema, mejorar la calidad de atención y permitir que los usuarios hagan uso adecuado del derecho otorgado por la ley.

\section{PALABRAS CLAVE: Cáncer cérvicouterino, reforma en salud, sistema de salud}

\section{SUMMARY}

Background: Cervical cancer (CC) is a disease included in the AUGE system for its high impact on the population. Within this system, every user has the right to be informed about its functioning to encourage timely care, however, at present the users' level of knowledge is unknown. Objective: To identify the degree of knowledge and sources of information on GES among women seeking care in the CC-AUGE program. Method: Cross-sectional study with a sample of 364 women, 237 with pre-invasive lesions and 127 with CC. Results: $18.7 \%$ of women reported knowledge of the system, $24.5 \%$ reported partial knowledge and $56.7 \%$ mentioned that they do not know about it. $62 \%$ of the women who knew the GES understood it as a system that gives financial protection and $45 \%$ understood that it covers serious or catastrophic illness. Regarding 
sources of information about GES, 71\% reported that received information by health staff or health services, $29 \%$ by mass media, $8 \%$ by neighbors and $8 \%$ from other sources. Conclusions: The degree of knowledge about the GES system is low among these women and it is associated mainly with financial protection and coverage of diseases. It is relevant to provide greater information that facilitates the efficient function of the system, quality of care, and to allow women to use the right given to them by the Chilean law.

\section{KEY WORDS: Cervical cancer, health reform, health system}

\section{INTRODUCCIÓN}

El sistema de salud chileno enfrenta una reforma en salud, donde ha sido incorporado el sistema de garantías explícitas en salud (GES) (1). El sistema GES refleja la distribución de recursos en salud para las patologías de mayor morbilidad y mortalidad en el país (2), en las que a su vez se establece prioridad en la atención reflejada en 4 garantías: acceso, calidad, oportunidad y protección financiera $(3,4,5)$.

El cáncer cérvicouterino (CC) es una de las patologías incluidas en el sistema GES debido a su alto impacto social y económico, y dado que afecta principalmente a mujeres cada vez más jóvenes y en edad reproductiva $(6,7,8)$. La evidencia muestra que la sobrevida para las pacientes con CC es alta en etapas tempranas de la enfermedad, siendo el diagnóstico precoz y el tratamiento oportuno, factores fundamentales en la evolución de esta patología (4).

En relación al sistema GES, todo usuario tiene derecho a ser informado sobre su funcionamiento, de manera que lo conozca y pueda ser atendido oportunamente (9). Sin embargo, pese a que se ha incrementado el nivel de conocimiento en los últimos años, aun existe desconocimiento por parte de los usuarios de FONASA (Fondo Nacional de Salud) e ISAPRES (Instituciones de Salud Previsional) sobre el funcionamiento de este sistema (10).

Específicamente en relación al programa $\mathrm{CC}$, no se encontraron estudios en la literatura que reporten el nivel de conocimientos de las usuarias, por lo que el identificar el grado conocimiento de la población es una herramienta fundamental para favorecer el acceso oportuno a la atención.

El objetivo del presente estudio es identificar el grado de conocimiento y las fuentes de información sobre el sistema GES que tienen las mujeres atendidas en el programa CC-AUGE (Acceso Universal de Garantías Explícitas) del Servicio de Salud Metropolitano Sur Oriente (SSMSO).

\section{PACIENTES Y MÉTODO}

Estudio descriptivo de corte transversal, realizado en un grupo de 364 mujeres atendidas en el programa CC-AUGE del SSMSO. La muestra está constituida por 237 mujeres con lesiones preinvasoras y 127 mujeres con CC. Las mujeres con lesiones preinvasoras, corresponden a una muestra probabilística con un intervalo de confianza del 95\%, representativa de las mujeres con lesiones preinvasoras ingresadas al programa CC-AUGE del SSMSO, y las mujeres con CC corresponden al total del universo de mujeres con cáncer ingresadas al programa y que se encontraban vivas al momento de la realización de la entrevista. Los criterios de inclusión fueron: tener diagnóstico confirmado por especialista de una lesión invasora o preinvasora de cuello uterino y haber ingresado al programa AUGE del SSMSO durante los años 2004 y 2005. Las mujeres que cumplieron con estos criterios fueron entrevistadas entre los meses de mayo y octubre del año 2006. Para la recolección de los datos, se llevó a cabo una entrevista en el domicilio de las mujeres, realizada por Enfermeras Matronas y Matronas, las que fueron previamente entrenadas para este propósito. Las variables a analizar en el presente estudio son: características de las mujeres (edad, años de escolaridad, previsión, presencia de infecciones de transmisión sexual, inicio de actividad sexual, número de parejas sexuales), motivo de ingreso al programa CC-AUGE (Papanicolaou alterado, cuello sospechoso, otro) y conocimiento del sistema GES. La variable conocimiento del sistema GES fue medida a través de tres preguntas: una pregunta cerrada (¿conoce usted el sistema de garantías explicitas del plan AUGE/GES? (SiNo-Parcialmente)), y dos preguntas abiertas (¿De qué se trata el sistema de garantías explícitas? / ¿Cómo se informó del sistema de garantías explícitas?). Los datos cuantitativos fueron analizados con el programa SPSS 16.0 con medidas de tendencia central, de dispersión y análisis de frecuencia. Para el análisis de las dos preguntas abiertas de conocimiento del sistema GES se utilizó análisis de contenido descrito por Krippendorf (11), generando una descripción temática de cada una de las respuestas entregadas. Las respuestas fueron registradas de manera textual de acuerdo a lo expresado por las mujeres. La unidad de análisis fue la frase que la 
mujer entregó frente a la pregunta correspondiente. Las ideas comunes fueron agrupadas en subtemas, los cuales dieron la base para el desarrollo de cada tema. Cada una de las autoras del presente artículo realizó un análisis de las respuestas entregadas por las mujeres llegando posteriormente a un consenso en los temas y subtemas. Los comités de ética de la Escuela de Enfermería de la Pontificia Universidad Católica de Chile y el del SSMSO aprobaron el estudio. Se solicitó consentimiento informado a cada una de las mujeres que participaron en este estudio.

\section{RESULTADOS}

Características de las mujeres: El promedio de edad de la muestra es de 42,2 \pm 12 años con un rango de edad entre 22 y 81 años. Los años de escolaridad promedio son de $9 \pm 3,5$ años. El $100 \%$ de las mujeres pertenece a FONASA, con un $79,1 \%$ de la muestra concentrada en los niveles $A$ y B. El $8,2 \%$ de las mujeres refiere tener antecedentes de infección de transmisión sexual. La edad de inicio de relaciones sexuales fue de 17,7 $\pm 3,5$ años. El promedio de parejas sexuales es de 2,6 \pm 2,6 parejas, con un rango de 1 pareja a 11 parejas sexuales.

Motivo de ingreso al programa CC-AUGE: El 83\% de las usuarias ingresó al programa CC-AUGE por un Papanicolaou alterado, un 6,6\% por presentar cuello sospechoso, el 4,9\% vía consulta de urgencia y el $5,5 \%$ otra causa (derivación del extra sistema por sangrado genital, dolor, u otros síntomas).

Conocimiento del sistema GES: Al analizar la pregunta ¿conoce usted el sistema de garantías explicitas del plan AUGE?, los resultados reflejan que el $18,7 \%(n=68)$ de las mujeres refiere conocer el sistema GES, el $24,5 \%(n=89)$ refiere que lo conoce parcialmente, mientras que el $56,7 \%(n=206)$ dice que no lo conoce.

Al preguntarles ¿de qué se trata el sistema de garantías explícitas?, los contenidos que emergen de los relatos de las personas que refieren conocer total o parcialmente el sistema GES $(n=157)$ se agrupan en cuatro grandes temas: sistema que otorga protección financiera, sistema que otorga cobertura de algunas enfermedades, sistema que es más eficiente para solucionar problemas y sistema que otorga beneficios a las personas. En la Tabla I, se presentan los temas, con sus respectivos subtemas y relatos de las usuarias.

Al preguntarles ¿cómo se informó del sistema de garantías explícitas?, las usuarias $(n=163)$ men- cionaron todas las posibles fuentes de información que confluyen en las siguientes categorías: personal de salud, medios de comunicación, vecinos o familiares, otros. La distribución de los temas con sus correspondientes relatos se presenta en la Tabla II.

\section{DISCUSIÓN}

La presente investigación, es la primera que reporta resultados del conocimiento que tienen respecto del sistema GES las personas atendidas en algunos de los programas AUGE, en este caso del programa CC del SSMSO.

El porcentaje de mujeres que refiere conocer el sistema GES es bajo en la presente muestra, si se considera que menos del $50 \%$ de la muestra señala conocerlo total o parcialmente. Estos resultados indican un déficit de conocimiento en este grupo de mujeres, el cual a su vez se ve limitado a solo alguno de los aspectos que considera el sistema GES.

Al comparar estos resultados con estudios realizados en el país sobre conocimientos del sistema GES, se hace más evidente la necesidad de seguir evaluando este aspecto con mayor detalle y de enfatizar en la necesidad de conocimiento sobre el sistema GES en distintos grupos de población. Lo anterior radica en la gran diferencia existente entre las cifras reportadas. Es así como en el año 2008, la Superintendencia de Salud presentó los resultados obtenidos de una encuesta de evaluación ciudadana (12) con un total de 1.447 entrevistados (sector público y privado), donde el $45 \%$ de los encuestados respondió conocer el sistema GES y un $28 \%$ refirió conocerlo parcialmente, señalando finalmente que un $73 \%$ de los entrevistados sabía algo del sistema de GES. En el año 2009 (13), en un total de 1.728 encuestados se reporta un aumento del porcentaje, llegando a un $78 \%$ con conocimiento del sistema GES ( $47 \%$ si lo conoce y un $31 \%$ parcialmente lo conoce). El porcentaje de conocimiento reportado en ambos estudios casi duplica el reportado por las mujeres en esta muestra. La diferencia en los resultados puede estar explicado por dos causas: que el presente estudio fue realizado dos años antes previo a los estudios citados anteriormente, y que los grupos poblacionales son diferentes, ya que los estudios referenciados fueron hechos en la población general, no perteneciente a algún programa AUGE especifico. Esto a su vez, nos abre una real interrogante respecto del verdadero conocimiento que tienen los usuarios, que actualmente están recibiendo atención en algún programa AUGE vigente. 


\section{Tabla I}

CONOCIMIENTO DE LAS MUJERES ENTREVISTADAS RESPECTO AL SISTEMA GES

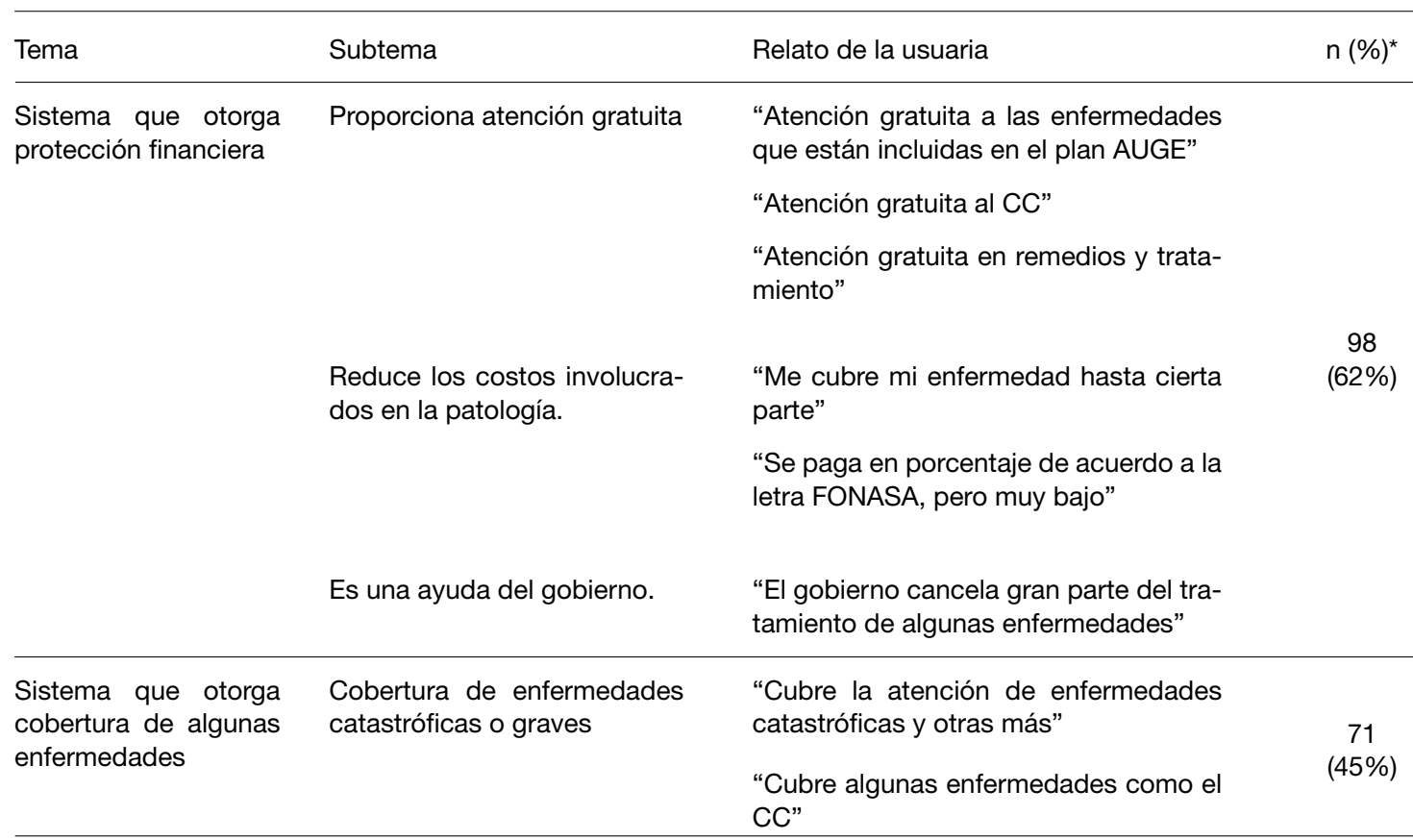

Sistema que es más eficiente para solucionar problemas de salud
Otorga atención preferencial

Otorga atención rápida

Otorga atención con plazos
“Atención preferencial, más rápido para los cánceres".

"La gente que tiene menos recursos tiene preferencia, al igual que la gente de 60 años, cuando uno tiene baja la FONASA (A ○ B)"

"Atención rápida porque es grave"

"Que hay plazos limitados para el seguimiento de las enfermedades"

"Las personas tienen derecho a la atención si concurre a un hospital"

sonas

*La suma de los porcentajes es superior al 100\%, debido a que las mujeres dieron más de una respuesta.

La necesidad de obtener información adecuada sobre el nivel de conocimiento por grupos de población, se ve reflejada además en otro estudio conducido por la Superintendencia de Salud (14) durante los años 2005-2006, en donde se evaluó el conocimiento del sistema de GES de beneficiarios de ambos subsistemas por separado con una muestra de 66 beneficiarios FONASA y 101 de ISAPRES. En los beneficiarios de FONASA el nivel de conocimiento fue menor que el de los beneficiarios del sistema privado, siendo de $56 \%$ versus un $98 \%$ en julio del año 2005 y $80 \%$ versus $92 \%$ en noviembre de 2006, respectivamente. Pese a que se observó un incremento en el conocimiento de los usuarios del sistema público, éstos siempre estuvieron más de 10 puntos porcentuales por debajo de los usuarios del sistema privado. Esto en parte puede respaldar el bajo porcentaje de la población que en el presente estudio refiere conocer el sistema de GES, dado que el $100 \%$ de la muestra estudiada pertenece a FONASA. Sin embargo, el porcentaje reportado en ambos periodos es superior al reportado en nuestro estudio. 
Tabla II

FUENTE DE INFORMACIÓN SOBRE EL SISTEMA GES

\begin{tabular}{|c|c|c|}
\hline Fuente de Información & Relato de la usuaria & $\mathrm{n}(\%)^{\star}$ \\
\hline \multirow{5}{*}{$\begin{array}{l}\text { Personal de salud } \\
\text { o por algún servicio } \\
\text { de salud } \\
\text { (consultorio u hospital) }\end{array}$} & "En el consultorio, explicación de la matrona y cartel informativo" & \multirow{5}{*}{$\begin{array}{c}117 \\
(71 \%)\end{array}$} \\
\hline & "El médico del CDT" & \\
\hline & "Folleto informativo del consultorio y en el trabajo" & \\
\hline & $\begin{array}{l}\text { "Fui informada por la matrona del servicio de Ginecología } \\
\text { Oncología" }\end{array}$ & \\
\hline & "Información en CDT en ventanilla" & \\
\hline \multirow[t]{4}{*}{ Medios de comunicación masiva } & "Lo escuché en las noticias y en todo lo que veo" & \multirow{4}{*}{$\begin{array}{c}47 \\
(29 \%)\end{array}$} \\
\hline & "Por televisión, diario" & \\
\hline & $\begin{array}{l}\text { "Se informa por televisión y diarios, en el servicio no lo } \\
\text { mencionaron" }\end{array}$ & \\
\hline & "Por las noticias" & \\
\hline \multirow[t]{2}{*}{ Vecinos o familiares } & "Por la vecina que tenía cáncer de mama" & \multirow{2}{*}{$\begin{array}{c}13 \\
(8 \%)\end{array}$} \\
\hline & "Por familiares y amigas" & \\
\hline \multirow[t]{4}{*}{ Otras fuentes de información } & "La niña que atiende en oficina AUGE" & \multirow{4}{*}{$\begin{array}{c}13 \\
(8 \%)\end{array}$} \\
\hline & "Teléfono de FONASA (número 600)" & \\
\hline & "Por folleto de FONASA" & \\
\hline & "Por capacitación en el trabajo" & \\
\hline
\end{tabular}

\footnotetext{
* La suma de los porcentajes es superior al 100\%, esto es debido a que las mujeres dieron más de una respuesta correspondiendo a diferentes temas. CDT: Centro de Diagnóstico y Tratamiento.
}

Aunque persisten diferencias al comparar el nivel de conocimientos de los usuarios de FONASA con el grado de conocimiento de las mujeres incluidas en este estudio, es importante señalar que ambos apuntan a la necesidad de información respecto del sistema de GES. Esto cobra aun mayor relevancia si se considera que el sistema GES es un derecho de salud otorgado a los usuarios por ley $(15,16)$. Los usuarios necesitan ser informados ya que así podrán conocer los mecanismos para su exigibilidad, recibir la atención que corresponde, y ser capaces de realizar reclamos en caso de que no reciban la atención correspondiente (15).

Respecto del análisis de lo que las mujeres refieren conocer al preguntarles por el sistema GES, la protección financiera y cobertura de enfermedades son los dos aspectos mayormente mencionados por las usuarias. Ambos aspectos fueron también reconocidos por usuarios de FONASA como las principales ventajas del sistema en el estudio realizado el año 2009 por la Superintendencia de Salud (17). Probablemente este conocimiento es el que mayoritariamente las usuarias manejan debi- do a que es considerado de mayor relevancia por ellas, o porque quizás ha sido el aspecto informado con mayor frecuencia al momento de ser diagnosticadas con una lesión invasora o preinvasora de cuello uterino. Otra posible causa puede ser que las usuarias al ser sometidas al copago en su atención según el tramo de FONASA, son informadas respecto de esta garantía, señalándoles que el resto será financiado por un subsidio público (3).

Respecto de las fuentes de información que reportan las usuarias, cabe señalar que coincide con un reporte de FONASA (9) donde el mayor porcentaje de información fue recibida por parte de los profesionales en los centros de salud.

El conocimiento de que disponen las usuarias debe ser considerado al momento de analizar la navegación de ellas por el sistema de salud, ya que podría afectar en una de las garantías conocida como de "acceso" al sistema AUGE propiamente tal. Es relevante mencionar que aunque las usuarias estén familiarizadas con la palabra AUGE o GES, y refieran conocer el sistema, esto no indica que dispongan o manejen la información suficiente 
y correcta que les permita identificar todos los aspectos que este sistema incluye, y por ende que les permita ser capaces de exigir adecuadamente los derechos establecidos por la ley (15). Al respecto, cabe destacar que la garantía de oportunidad, una de las 4 GES, fue solo referenciada por el $5,7 \%$ de la muestra en el presente estudio, lo que demuestra que aspectos con mayor detalle sobre el sistema GES son de conocimiento deficiente en este grupo de mujeres. Estudios previos del MINSAL mencionados anteriormente $(4,13,14)$, confirman el inadecuado conocimiento de los usuarios de este aspecto y de otros como son el reconocimiento de que AUGE y GES son términos equivalentes, cuáles y cuántos son los problemas de salud incluidos, el conocimiento de sus deberes y beneficios y el conocimiento sobre el acceso, oportunidad y calidad de las GES.

\section{CONCLUSIÓN}

Podemos concluir que existe una necesidad de mejorar la entrega de información a las usuarias del sistema CC-AUGE, para de esta manera fortalecer el funcionamiento eficiente de este sistema, mejorar la calidad de atención a este grupo de estudio y también permitir que los usuarios hagan uso adecuado del derecho otorgado por ley en nuestro país.

\section{BIBLIOGRAFÍA}

1. De Azevedo AC, Mardones-Restat F. The essential health reform in Chile; a reflection on the 1952 process. Salud Publica Mex 2006;48:504-511

2. CEPAL [Sede Web]. Protección social y sistemas de salud. La protección social: de cara al futuro, acceso, financiamiento y solidaridad, 2006. [acceso 18 de Julio de 2009]. Disponible en: http://www.eclac.org/ celade/noticias/paginas/5/27255/Cruces2.pdf.

3. Superintendencia de Salud [Sede Web]. Qué es el Auge; 2009. [acceso 11 de Agosto de 2009]. Disponible en: http://www.supersalud.cl/568/propertyvalue-800.html.

4. Román O, Muñoz F. Una mirada crítica en torno al plan AUGE. Algunos aspectos generales y valóricos. Rev Méd Chile 2008;136:1599-1603 .

5. Vergara-Iturriaga M, Martínez-Gutiérrez MS. Financiamiento del sistema de salud chileno. Salud Pública Mex 2006;48:512-21.

6. MINSAL [Sede Web]. Garantías explícitas en salud: Guía clínica del cáncer cervicouterino, 2005. [acceso 8 de Junio de 2009]. Disponible en: http://www.minsal.cl.

7. Waggoner SE. Cervical cancer. Lancet2003;361:221725

8. Montes L, Mullins M, Urrutia MT. Calidad de vida en mujeres con cáncer cérvico uterino. Rev Chil Obstet Ginecol;2006;71:129-34.

9. FONASA [Sede Web]. Todo usuario del sistema público de salud tiene derecho a que se le entregue orientación y/o información sobre el funcionamiento de los establecimientos a los que asista. [acceso 2 de Marzo de 2010]. Disponible en:

http://www.fonasa.cl/prontus_fonasa/antialone. html?page=http://www.fonasa.cl/prontus_fonasa/site/ artic/20041125/pags/20041125124817.html

10. Superintendencia de Salud [Sede Web]. Más de $8 \mathrm{mi}-$ llones de personas beneficiadas con el AUGE en 4 años. [acceso 6 de Septiembre de 2009]. Disponible en: http://www.supersalud.cl/568/fo-article-5389.pdf

11. Krippendorf K. Content Analysis. An introduction to its methodology. Thousands Oaks: Sage Publications 2004.

12. MINSAL [Sede Web]. AUGE 2005-2008: Implementación de las garantías explicitas en salud, 2008. [acceso 10 de Agosto de 2009]. Disponible en: http://www. supersalud.cl/568/articles-4325_recurso_1.pdf.

13. Superintendencia de Salud [Sede Web]. Conocimiento de la Reforma GES-AUGE y posicionamiento de la Superintendencia de Salud, 2009. [acceso 6 de Septiembre de 2009]. Disponible en: http://www.supersalud.cl/documentacion/569/articles-5353_recurso_1. pdf.

14. Superintendencia de Salud [Sede Web]. Resultado encuesta de otorgamiento de las Garantías explicitas en Salud: Verificación de Beneficiarios que Acceden a las GES III Etapa, 2006. [acceso 4 de Octubre de 2009]. Disponible en: http://www.supersalud.cl/568/ article-2844.html.

15. MINSAL. Hacia un nuevo modelo de gestión en salud. Contenidos del proyecto de ley de autoridad sanita $\neg$ ria y gestión en salud y de medidas administrativas inmediatas, 2002. [Acceso: 10 de marzo de 2009] Disponible en: http://www.ias.uchile.cl/files/biblioteca/gestion. pdf.

16. Clínicas de Chile A.G. [Sede Web]. Ampliación de la cobertura del plan AUGE: reflexiones sobre la eficiencia, 2008 [acceso 4 de Agosto de 2009]. Disponible en: http://www.clinicasdechile.cl/Coyuntura_11.pdf.

17. Superintendencia de Salud [Sede Web]. Informe nacional: Conocimiento de nuestros usuarios sobre GES. Plataforma de atención preferencial. 2008 [acceso 10 de Agosto de 2009]. Disponible en: http:// www.supersalud.cl/568/articles-3257_recurso_9.pdf. 